\title{
Immune analysis of lymph nodes in relation to the presence or absence of tumor infiltrating lymphocytes in triple-negative breast cancer
}

\author{
Ángela Quintana ${ }^{\mathrm{a}, \mathrm{b}}$, Vicente Peg ${ }^{\mathrm{b}, \mathrm{c}, \mathrm{d}}$, Aleix Prat ${ }^{\mathrm{e}, \mathrm{f}}$, Teresa Moliné ${ }^{\mathrm{c}}$, \\ Guillermo Villacampa ${ }^{\mathrm{g}}$, Laia Paré ${ }^{\mathrm{f}}$, Patricia Galván ${ }^{\mathrm{e}, \mathrm{f}}$, \\ Rodrigo Dientsmann ${ }^{\mathrm{g}}$, Peter Schmid ${ }^{\mathrm{h}}$, Giuseppe Curigliano ${ }^{\mathrm{i}}$, \\ Eva Muñoz-Couselo ${ }^{\mathrm{a}, \mathrm{j}}$, José Perez-García ${ }^{\mathrm{k}}$, Merce Marti ${ }^{\mathrm{b}}$, \\ Juan Blanco-Heredia ${ }^{1}$, Carla dos Anjos ${ }^{1}$, Miguel Vazquez ${ }^{\mathrm{m}}$, \\ Leticia De Mattos-Arruda ${ }^{1, * *}$, Javier Cortés ${ }^{a, k, n, o, *}$
}

a Vall D'Hebrón Institute of Oncology, Barcelona, Spain

${ }^{\mathrm{b}}$ Universidad Autónoma de Barcelona, Barcelona, Spain

' Department of Pathology, Vall D'Hebron University Hospital, Barcelona, Spain

${ }^{\mathrm{d}}$ Spanish Biomedical Research Network Centre in Oncology (CIBERONC), Madrid, Spain

${ }^{e}$ Medical Oncology Hospital Clinic, Barcelona, Spain

${ }^{\mathrm{f}}$ Translational Genomics and Targeted Therapeutics Group, IDIBAPS, Barcelona, Spain

${ }^{g}$ Oncology Data Science (ODysSey Group), Vall D'Hebron Institute of Oncology, Barcelona, Spain

${ }^{\mathrm{h}}$ Barts Cancer Institute, Queen Mary University London, United Kingdom

${ }^{\mathrm{i}}$ European Institute of Oncology, IRCCS, And University of Milano, Milan, Italy

${ }^{\mathrm{j}}$ Medical Oncology Hospital Vall D'Hebrón, Barcelona, Spain

${ }^{\mathrm{k}}$ IOB Institute of Oncology, Quironsalud Group, Barcelona, Spain

${ }^{1}$ IrsiCaixa, Hospital Universitari Trias I Pujol, Badalona, Spain

${ }^{\mathrm{m}}$ Barcelona Supercomputing Center, Barcelona, Spain

${ }^{\mathrm{n}}$ IOB Institute of Oncology, Quironsalud Group, Madrid, Spain

${ }^{\circ}$ Medica Scientia Innovation Research (MedSIR), Barcelona, Spain

Received 10 December 2020; received in revised form 26 January 2021; accepted 28 January 2021

\section{KEYWORDS}

Triple-negative breast cancer;

\begin{abstract}
Triple-negative breast cancer (TNBC) is a subtype of breast cancer with unmet medical needs. Several studies have proved that high levels of tumor infiltrating lymphocytes (TILs) at diagnosis of TNBC confer better prognosis and patients respond better to specific chemotherapies. Nonetheless, current evidence suggests that only $15 \%$ of TNBC patients have
\end{abstract}

\footnotetext{
* Corresponding author: Vall d'Hebrón Institute of Oncology, Paseo de Vall d'Hebrón 119-129, 08035 Barcelona, Spain. ** Corresponding author: IrsiCaixa, Hospital Germans Trias i Pujol. Ctra del Canyet, s/n. 08916 Badalona, Barcelona, Spain.

E-mail address: 1demattos@irsicaixa.es (L. De Mattos-Arruda), jacortes@vhio.net (J. Cortés).
} 
Tumor infiltrating lymphocytes; Lymph nodes; Neoantigens; Immune checkpoint inhibitors very high levels of TILs, and another 15\% lacks TILs. One possible reason to explain why patients have low TILs at diagnosis is that lymphocytes might be deactivated by an immune checkpoint in local lymph nodes, provoking their retention in there as they are unresponsive to other immune stimuli.

We have identified 15 high TILs ( $\geq 50 \%)$ and 20 low TILs $(\leq 5 \%)$ TNBC patients with localised tumour (T1c-T2N0M0) and compared the protein expression of five immune checkpoints in lymph nodes. We have also performed a customised 50-immune gene NanoString expression panel, the NanoString 360 Breast Cancer panel, and whole exome sequencing for mutation and neoantigen load analyses.

In low TILs, we observed higher expression of CTLA-4 in local lymph nodes, which could explain why lymphocytes get retained in there and do not migrate to tumour. These patients have also higher neoantigen load and higher expression of B7.H3 and B7.H4 in the tumour. In high TILs, we observed more PD-L1+ tumour cells and more expanded humoral response. These results could provide a strategy to revert low tumour immune infiltration at diagnosis of TNBC, improving their prognosis.

(c) 2021 Elsevier Ltd. All rights reserved.

\section{Introduction}

Triple-negative breast cancer (TNBC) is a poor prognosis breast cancer subtype, with chemotherapy being the backbone of treatment. However, over the last years, immune checkpoint inhibitors have demonstrated to play a significant role in the treatment of metastatic TNBC. Atezolizumab (an anti-PD-L1 monoclonal antibody) and pembrolizumab (an anti-PD-1 monoclonal antibody) have shown to improve progressionfree survival in patients with PD-L1 positive tumours $[1-3]$. In early TNBC, pembrolizumab added to neoadjuvant carboplatin and taxane-based therapy has shown a significant improvement in pathological complete responses, irrespective of PD-L1 expression [4]. Thus, PD-L1 is a predictive biomarker used to select immune-based treatment in patients with metastatic TNBC, but not in the early setting.

Tumor infiltrating lymphocytes (TILs) is one of the strongest potential biomarkers for TNBC patients. The first studies usually established a cut-off of $50 \%$ or higher $[5,6]$ as a good prognosis marker, but a recent pooled analysis published in 2019 [7] demonstrated a great benefit with TILs $\geq 30 \%$ in localised, node-negative TNBC. Many studies have proved that the presence of TILs predicts response to neoadjuvant [7-11] treatments, and that patients have better prognosis in terms of higher PFS and OS [5-13]. Unfortunately, only 15\% of TNBC patients present $\geq 50 \%$ of TILs at diagnosis, and around 15\% lacks TILs [14].

Lymph nodes (LN) are secondary lymphoid organs of the immune system. They are removed during breast cancer surgery to study for potential metastasis, as they are usually the first place of tumour dissemination. Their function is to provide a specific immune response to infections or tumour growth that occur in the surrounding tissues. Only a few researchers have studied the immune cell population in LN of breast cancer patients and correlated with prognosis [15-18]. One of these studies, done by Kohrt et al. [15], observed a decrease of CD4 and CD8 and an increase of CD1a in tumour-free axillary and sentinel $\mathrm{LN}$ of breast cancer patients in comparison to controls, and that increased levels of axillary CD4 T cell and CD1a dendritic cell population levels highly correlated with better diseasefree survival.

However, why some breast tumours are infiltrated by immune cells while others do not is not well known. Understanding this lack of tumour immune infiltration in some patients at the time TNBC is diagnosed might be of great help to optimise therapeutic strategies. In this study, we hypothesised that lymphocytes could be deactivated or not fully activated at the tumour-draining LN by one or several immune checkpoints, provoking a retention of anergic lymphocytes because they are unable to migrate to the tumour. In addition, we explored gene expression and mutational and neoantigen load status in these patients.

\section{Methods}

\subsection{Patients and samples}

Samples and data from patients included in this study were obtained from the Pathological Anatomy Department of Vall d'Hebrón Hospital and the BioBank Hospital Ramón y Cajal-IRYCIS (PT13/0010/0002), integrated in the Spanish National Biobanks Network, and they were processed following standard operating procedures with the appropriate approval of the Ethical and Scientific Committees. The inclusion criteria were TNBC patients with localised tumours $(>10$ and $\leq 50 \mathrm{~mm}$ ) with no $\mathrm{LN}$ or other organ affected (T1c/ T2N0M0), who did not receive neoadjuvant treatment. 
The exclusion criteria were patients with intermediate TILs $(>5 \%$ and $<50 \%$, scored following the latest published guidelines [19]), and the unavailability of tumours or LNs in formalin-fixed paraffin-embedded (FFPE). Why we decided to select more than 50\% compared to lower than $5 \%$ was to include both extremes from the TILs spectrum (something like enriched versus null TILs). In patients who underwent lymphadenectomy, we chose a LN from the first level with the largest number of germinal centres (GCs). We consider that the most active LN was the closest to the tumour, and counting GCs was a simple approach to measure immune activity. Informed consent was obtained only in patients whose surgery was performed after 2007 or when a blood sample was needed for whole exome sequencing (WES), according to local institutional review board requirements.

We cut slides from tumour and LN for immunohistochemistry (IHC) and gene expression. For WES, we selected tumour samples of all patients, and asked for a fresh blood sample as a normal control. For patients unable to give a blood sample, we used their LN because it did not have metastases. We assessed the quality and quantity of DNA to evaluate patients' eligibility for this test, and only 13 patients of each group were included.

\subsection{Immunohistochemistry}

We used the following antibodies: CTLA-4 (F-8, Santa Cruz Biotechnology, dilution 1/75), PD-1 (NAT105, Roche-Ventana, prediluted), PD-L1 (SP263, RocheVentana, prediluted), PD-L2 (D7U8C, CellSignaling Technology, dilution 1/50), OX-40 (H-10, Santa Cruz Biotechnology, dilution 1/500) and CD8 (SP57, RocheVentana, prediluted). PD-L2, CTLA-4 and OX-40 were run in Benchmark XT platform using UltraView Universal DAB (Ref: 760-500); CD8 in BenchMark ULTRA using UltraView Universal DAB (Ref: 760-500) de Ventana Medical Systems; and PD-1 and PD-L1 in Benchmark XT using OptiView DAB IHC Detection Kit (Ref: 760-700). We used OptiView Amplification Kit for CD8 and PD-L1 to facilitate the assessment. All kits and platforms were from Ventana Medical Systems.

In LN, wherever possible, we tried to score only lymphocytes when we were able to distinguish their staining from other cells. CTLA-4 distinctively stains lymphocytes (surface membrane) from macrophages (cytoplasmic), and PD-1 only stains the surface of lymphocytes, so in these two biomarkers, we only scored lymphocytes. For PD-L1, PD-L2 and OX-40, it was not possible to distinguish the different cells, so we scored the LN as a whole. For the assessment of GCs, we did a mean of different percentages for those LN that had several GCs. We did not include in the analysis LN with no GC, to distinguish those GCs with no stain (scored $0 \%)$.
In tumour, all antibodies stained the immune infiltrate, but only PD-L1 and OX-40 stained tumour cells-with the exception of two high TILs samples with PD-L2 tumour stain. Since the difference between two groups was precisely the presence of immune infiltrate, we scored exclusively tumour cells to compare those two antibodies. In the high TILs group, we also scored PDL1 in the immune infiltrate to compare as a binary variable. The cut-off for PD-L1+ was $\geq 1 \%$ in tumour cells, and $\geq 10 \%$ in immune infiltrate, following the same methodology as O. Tawfik et al., that used the SP263 PD-L1 clone [20].

\subsection{Statistical analysis}

A descriptive analysis of the variables included in the study was performed. Continuous variables were expressed as median and range, and categorical variables were expressed as absolute values and percentages.

Non-parametric methods were used to compare expression levels after rejecting the null hypothesis of normality with the Shapiro-Wilk test. The Mann-Whitney and the Fisher's exact tests were used for pairwise comparison. Data analysis was carried out using R statistical software, version 3.5.1.

\subsection{Gene expression by NanoString}

We extracted RNA using Maxwell $^{\circledR} 16$ LEV RNA FFPE kit (Promega), and prepared the samples following the $n$ Counter XT CodeSet Gene Expression protocol. We run the samples in two NanoString panels: a customised 50immune panel in tumour and LN (see Table S1 for the list of genes), and the 360 Breast Cancer panel [21] only in tumour. We analysed the data using Student $t$ test and Pearson's $\chi 2$ or analysis of variance (ANOVA) for the association between two variables. To identify genes whose expression was statistically significant, we used significance of microarrays (SAM) test with false discovery rate $<5 \%$ to compare between the two groups. We used software R, version 3.5.1, for the analysis.

\subsection{Whole exome sequencing}

For mutation calling, we selected MuTect2, which is a somatic single nucleotide polymorphism (SNP) and indel caller that combines the DREAM challengewinning somatic genotyping engine of the original MuTect [22] with the assembly-based machinery of Haplotype Caller (Call germline SNPs and indels via local re-assembly of haplotypes).

For neoantigen prediction, we used pVACseq, which is a cancer immunotherapy pipeline for the identification of personalised Variant Antigens by Cancer Sequencing using netMHCpan algorithm [23,24]. NetMHCpan predicts binding of peptides to any major histocompatibility complex (MHC) molecule of known sequence 
using artificial neural networks. The method was trained on a combination of more than 180,000 quantitative binding data and mass spectrometry (MS) derived MHC-eluted ligands. The binding affinity data covers 172 MHC molecules from human. The MS-eluted ligand data cover 55 human leucocyte antigens (HLA) and mouse allele and is able to analyse peptides of any length.

\section{Results}

A total of 787 pathology reports of TNBC patients between 1998 and 2009 were identified at Hospital Vall d'Hebrón in Barcelona, and Hospital Ramón y Cajal in Madrid, Spain. Patients diagnosed after 2010 did not have $\mathrm{LN}$ available in paraffin blocks, because both hospitals implemented one-step nucleic acid amplification (OSNA) for the assessment of LN metastasis in primary surgeries and, therefore, they were ineligible for further inclusion.

As described in Fig. 1a, we included 35 patients in the study, 15 high TILs $(\geq 50 \%)$ and 20 low TILs $(\leq 5 \%)$. We excluded patients with intermediate TILs to better discriminate patients with high versuslow TILs. Based on their histology, high TIL patients had immune inflamed phenotype; for low TILs cohort, we considered patients with both the immune excluded and dessert phenotypes. Patient characteristics were similar in both groups, although age was slightly lower in the high TILs cohort compared with the low TILs (median age, 55 versus 61 years, respectively; Table 1). We followed the study workflow described in Fig. 1b, in which we could only include 13 patients of each group for WES.

All samples were classified as basal subtype by PAM50 signature, except two low TILs that were considered luminal A, which is probably anecdotal. By TNBC signature, 15 of 15 high TIL patients were basallike immune activated (BLIA); however, low TILs were more heterogeneous: 9 of 18 were BLIA, 1 of 18 was basal-like immune suppressed (BLIS), 4 of 18 were luminal/androgen receptor (LAR) and 4 of 18 were mesenchymal (MES); 2 patients were unable to be analysed. It is interesting to highlight that were some BLIA among the low TIL tumours, even though they have very low counts of TILs.

\subsection{CTLA-4 is more expressed in lymphocytes from LNs of low TIL patients}

A higher expression of CTLA-4 by IHC in axillary lymphocytes was observed in patients with low TILs

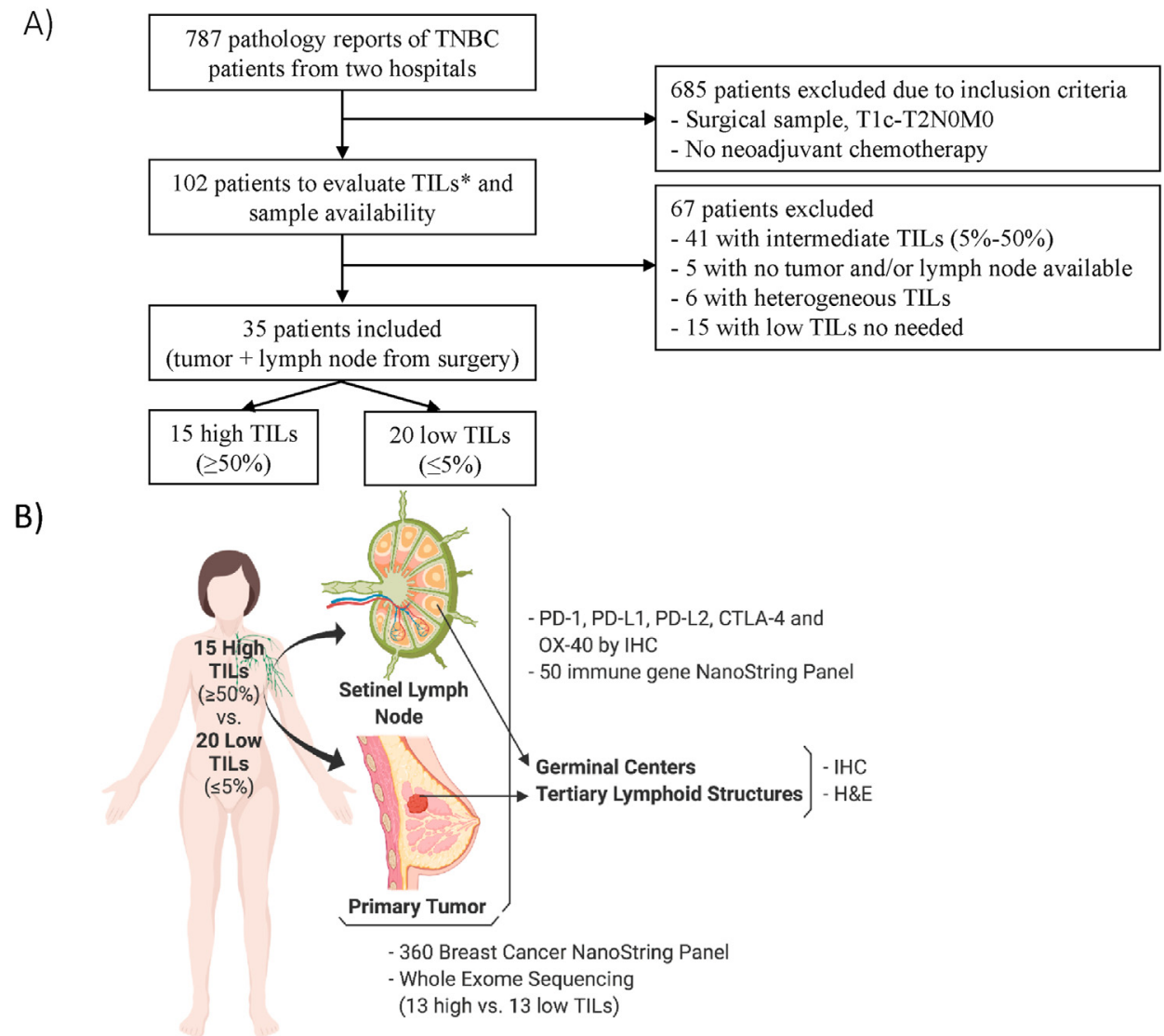

Fig. 1. Study schematics. a) Samples; b) methods. H\&E, haematoxylin and eosin; IHC, immunohistochemistry; TIL, tumor infiltrating lymphocytes; TNBC, triple-negative breast cancer. 
Table 1

Patient's clinical and anatomopathological characteristics.

\begin{tabular}{lll}
\hline Variables & $\begin{array}{l}\text { High TILs }(\geq 50 \%) \\
15 \text { patients }\end{array}$ & $\begin{array}{l}\text { Low TILs }(\leq 5 \%) 20 \\
\text { patients }\end{array}$ \\
\hline $\begin{array}{c}\text { Age at diagnosis (years) } \\
\text { Median (range) }\end{array}$ & $56(26-79)$ & $62(38-79)$ \\
$\begin{array}{c}\text { Type of surgery (\%) } \\
\text { Tumourectomy }\end{array}$ & $9(60 \%)$ & $16(80 \%)$ \\
Mastectomy & $6(40 \%)$ & $4(20 \%)$ \\
Tumour size (mm) & & \\
Mean & 22,5 & 20,1 \\
Median & 22 & 18 \\
Range & $11-50$ & $11-35$ \\
TNM (\%) & & \\
T1cN0M0 & $6(40 \%)$ & $12(60 \%)$ \\
T2N0M0 & $9(60 \%)$ & $8(40 \%)$ \\
Histology (\%) & & $15(75 \%)$ \\
Invasive ductal & $12(80 \%)$ & -1 \\
$\quad$ G2 & -0 & -14 \\
G3 & -12 & $0(0 \%)$ \\
Medullary G3 & $3(20 \%)$ & $2(10 \%)$ \\
Metaplastic G3 & $0(0 \%)$ & $1(5 \%)$ \\
Mucinous & $0(0 \%)$ & $1(5 \%)$ \\
Lobular G2 & $0(0 \%)$ & $1(5 \%)$ \\
Undifferentiated G3 & $0(0 \%)$ & $5(25 \%)$ \\
Lymph node resection (\%) & & $15(75 \%)$ \\
Lymphadenectomy & $9(60 \%)$ & \\
Selection of Sentinel & $6(40 \%)$ & \\
lymph/s node/s & & \\
\hline
\end{tabular}

TIL, tumor infiltrating lymphocytes; TNM, tumour-node-metastasis.

status, in comparison to that of high TILs (median 7\% versus $4 \%$, respectively, $p=0.01$ ); OX-40 staining in the whole LN was higher in low TILs (median 30\% versus $15 \%$, respectively, $\mathrm{p}<0.01$ ) (Fig. 2a). PD-1, PD-L1 and PD-L2 comparisons were not statistically significant, but PD-L2 showed a trend to be more expressed in low TILs group; the vast majority in antigen presenting cells of the paracortical area.

We designed a customised NanoString gene expression panel with the 50 most important markers for the study (Table S1). When we run this panel in the LN samples, they clustered in two groups, but it was independently of the immune infiltration of the matching primary tumour (data not shown). With SAM analysis, we observed only two genes differentially more expressed in LN of high TILs patients: CD68 ( $\mathrm{t}$ score 1.72) and CD8A (t score 1.28). However, by ANOVA test, CD68 and CD4 (but not CD8A) reached statistical significance $(\mathrm{p}=0.01$ and 0.03 , respectively). CD8A and $\mathrm{CD} 3$ showed a trend to have more expression in LN of high TILs ( $p=0.054$ and 0.07 , respectively). None of the genes highly expressed in LN of low TILs patients were statistically significant.

\subsection{High TIL patients have more tertiary lymphoid structures and more and larger germinal centres}

We then evaluated the five biomarkers in GCs by IHC, and the presence of tertiary lymphoid structures (TLSs) in tumour haematoxylin and eosin slides to study the humoral response in patients of both groups. We performed IHC comparison of the GCs between the two groups, which are shown in Fig. S1.

There were differences in shape, number and size of GCs between patients. In both groups, we found wellformed (circular) and irregular GCs, being this last usually associated with impaired humoral responses. To assess the quantity, based on the number of GCs observed in all samples, we considered $\leq 3$ GCs as a limited humoral response and $\leq 100 \mu \mathrm{m}$ (mean diameter) as a small GC. We observed that high TILs patients usually had more and larger GCs than low TILs patients (median $80 \%$ versus $35 \%, \mathrm{p}=0.02$ ). However, with the 50-immune gene expression panel, we did not see any B cell markers differentially expressed between patients.

In tumour, we observed almost twice as many patients with TLS in high TILs group than in low (47\% versus $25 \%, p=0.28)$. TLS of high TILs patients were usually more numerous and larger, whereas low TILs patients had very few-one or two at the most-and smaller. Most of them were extratumoural (at the periphery of the tumour area), but in some high TILs patients, they were present within the tumour.

\subsection{High TIL patients have higher presence of $P D-L 1+$ tumour cells but lower number of neoantigens}

We observed that all five biomarkers stained immune cells but only PD-L1 and OX-40 stained tumour cells (with the exception of two samples PD-L2+ in tumour cells). Therefore, they were the only ones comparable between high and low TILs samples. PD-L1 was more expressed in tumour cells of high TILs (median $0.0 \%$ versus $0.5 \%$, respectively, $\mathrm{p}<0.001$ ) and $\mathrm{OX}-40$ in tumour cells of low TILs (median 35\% versus 15\%, respectively, $\mathrm{p}<0.01$ ) (Fig. 2b). All high TILs patients showed PD-L1 staining in immune cells (median $15 \%[3 \%-60 \%])$.

When we examined the staining of the different antibodies in the same area, we observed that, in high TILs patients, PD-L1 and OX-40 usually stained intratumoural and stromal lymphocytes adjacent to the tumour (Fig. $3 \mathrm{a}-\mathrm{c}$ ). Using CD8 to identify cytotoxic T lymphocytes, we observed that CD8 staining overlapped with PD-L1 and OX-40 (Fig. 3d). In these patients, we also performed a binary correlation of PD-L1 between tumour cells (PD-L1+ TC $\geq 1 \%$ ) and immune cells (PD$\mathrm{L} 1+\mathrm{IC} \geq 10 \%$ ). This was statistically significant ( $\mathrm{p}$ < 0.01 ), in which we did not observe any patient who were PD-L1+ in tumour cells and PD-L1- in the immune infiltrate.

We noticed that tumour samples clustered almost perfectly in two groups depending on the presence of TILs when we run the NanoString 50-immune panel. Using SAM analysis, we discovered that B7.H4, B7.H3 (CD276), VEGF, CD73 and CD56 were overexpressed 
A)
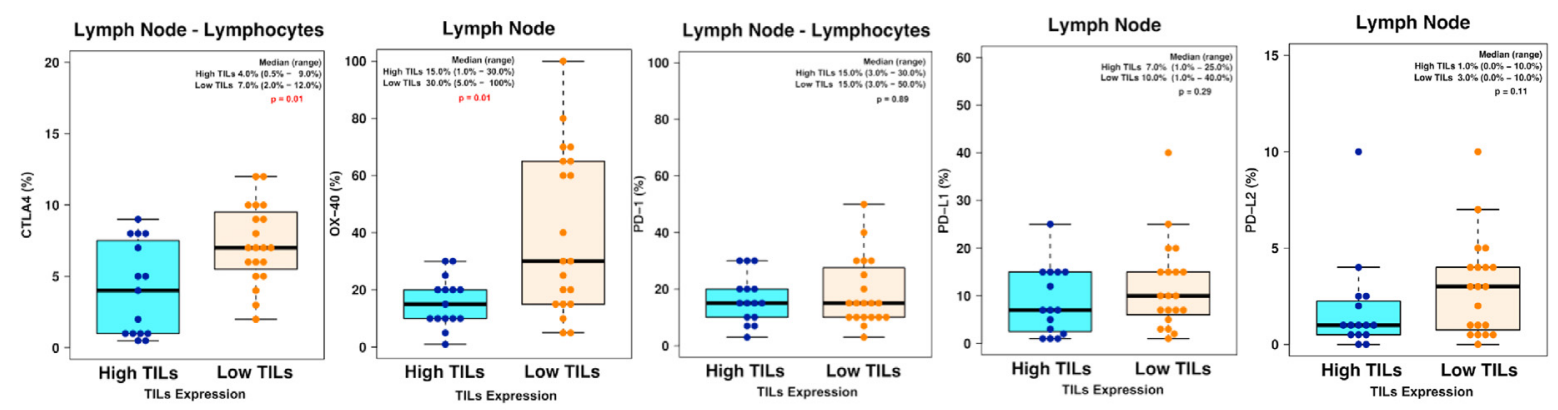

B)
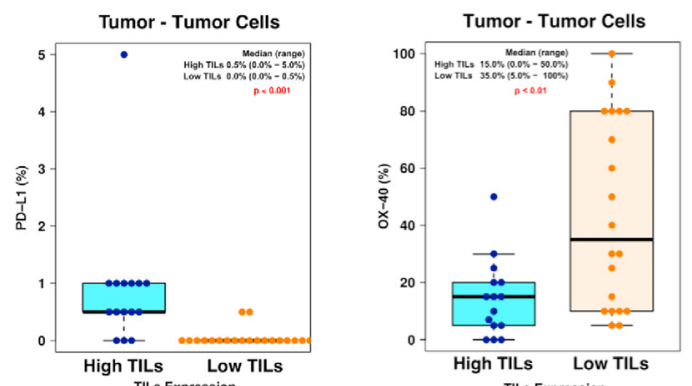

Fig. 2. Box plots comparing, between high and low TILs, the expression of: a) CTLA-4 (only lymphocytes), OX-40; PD-1 (only lymphocytes), PD-L1 and PD-L2 in lymph nodes; b) PD-L1 and OX4 in tumour cells of the primary tumour.

A) PD-L1 (4x)

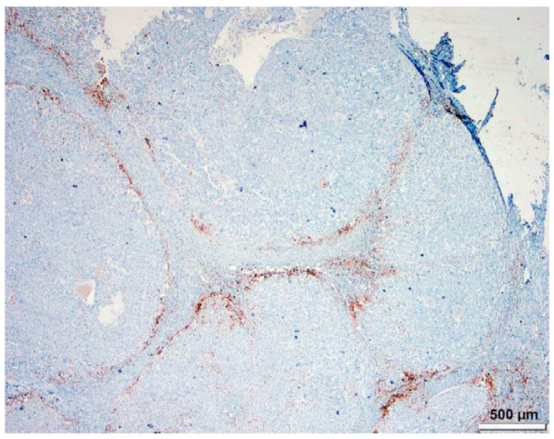

C) $\mathrm{OX}-40(20 \mathrm{x})$

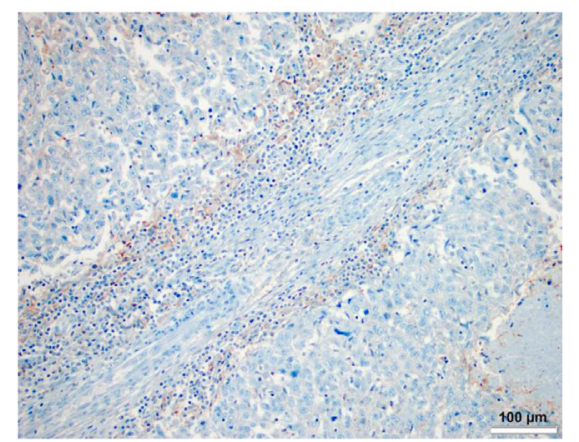

B) PD-L1 (20x)

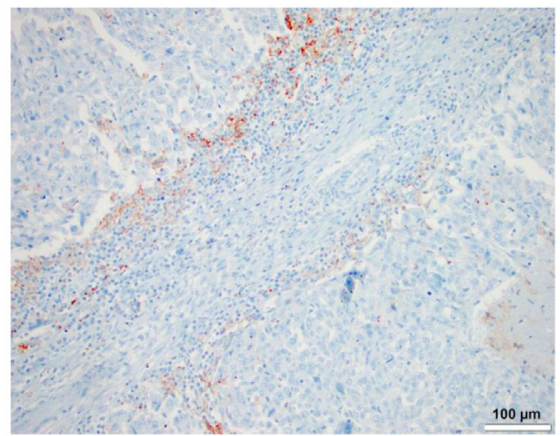

D) $\operatorname{CD8}(20 \mathrm{x})$

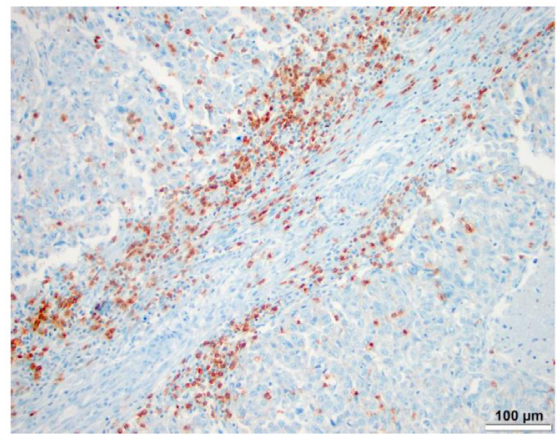

Fig. 3. Tumour sample of a high TILs patient stained with PD-L1, CD8 and OX-40. a) PD-L1 staining in tumour cells (4×); b) detail of PD-L1 staining in TILs $(20 \times)$; c) OX-40 $(20 \times)$; d) CD8 $(20 \times)$. 
A)

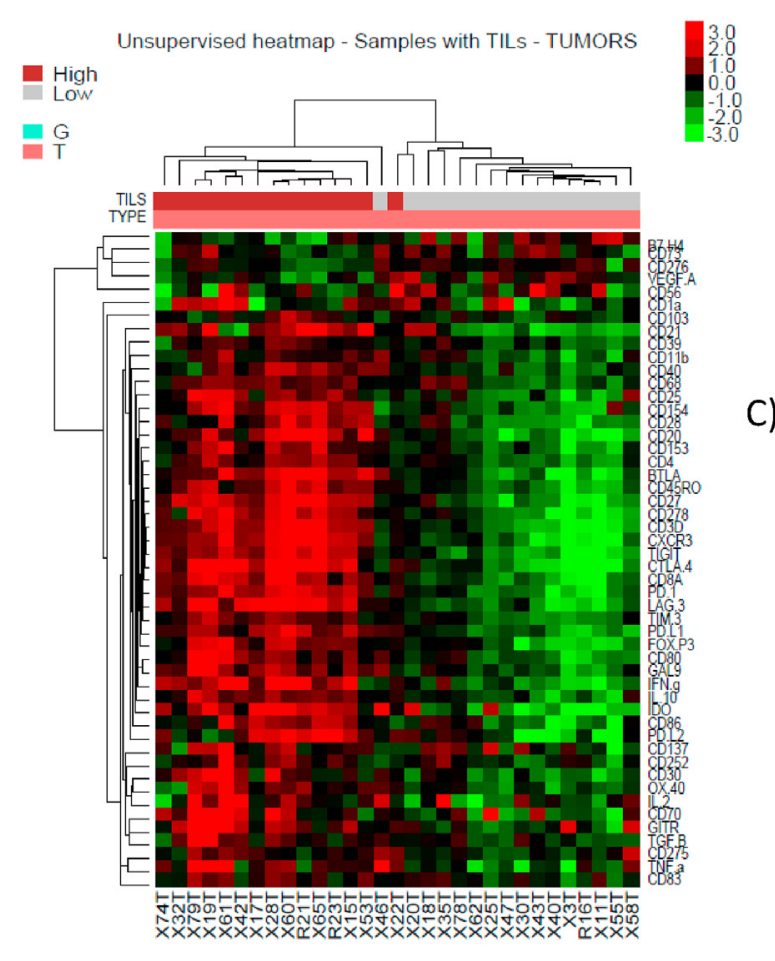

B)

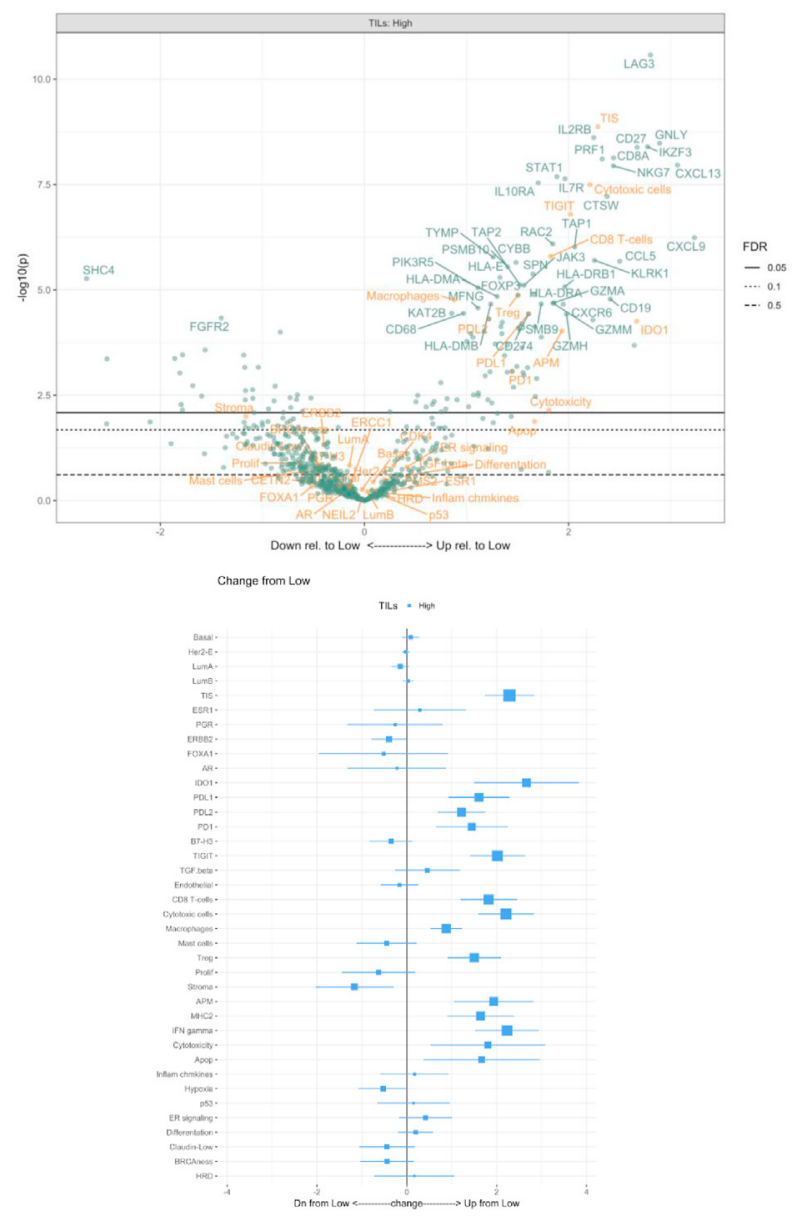

Fig. 4. NanoString results of the two panels. a) SAM analysis of the 50-immune gene panel run in tumour samples; b) volcano plot of the Breast Cancer 360 gene panel in high TILs patients compare to low; c) Forest Plot of the Breast Cancer 360 gene panel in high TILs patients compare to low. FDR, false discovery rate.

in low TILs, and all the other genes were more expressed in high TILs patients, as shown in the heatmap of Fig. 4a. By ANOVA analysis, only the first three reached statistically significance. We also realised that, unlike other immune checkpoint inhibitors, B7.H4 and B7.H3 were statistically more expressed in tumour in comparison with LN (data not shown).

When we run the NanoString 360 Breast cancer panel, we could observe that most of the immune signatures were significantly more expressed in high TILs patients: Tumor Inflammation Signature (TIS), Antigen Presenting Machinery (APM) and several immune checkpoint inhibitors (LAG3, PD-1, PD-L1/2, IDO1), among others (Fig. 4b). Non-classical HLA class I HLA-E, a common altered HLA phenotype in tumours, was also more expressed in high TILs. Although the forest plot showed a trend of TGF-beta signature to be more expressed in the tumour of high TILs, we noticed that low TILs had several TGF-beta signature genes significantly more expressed in the SAM analysis (TGFB2, BMPR1B, COMP, THBS2, THBS4, BAMBI, ID4, INHBB and FST) and none from this signature was downregulated in these patients. The customised 50immune panel only had TGFB1, and it was significantly more expressed in LNs than tumours, and in high TIL tumours in comparison with low TIL tumours. No differences were seen between the LNs of the two groups. In low TIL samples, only stromal signature was significantly more expressed. The upregulated genes were KRT14 and KRT17 (keratin); COL11A1 and COL9A3 (collagen); COMP, ASPN and VCAN (extracellular matrix proteins); and CLDN1, SPP1, LAMB3, CDH2, SNAI2 and ITGB3 (cell to cell, and cell to extracellular matrix adhesion), which probably increase the stiffness of the stroma. We only found FGFR2 (fibroblast growth factor receptor 2), a gene directly related to fibroblasts, to be overexpressed in low TILs. Other signatures like hypoxia, proliferation and mast cell showed a tendency to more expression (Fig. 4c).

We then proceeded to sequence the tumour samples to investigate the mutation and neoantigen load. We identified 519 non-synonymous mutations with the potential to generate neoantigens, 50 of which are displayed in Fig. 5a. Mean neoantigen per sample was 
A)
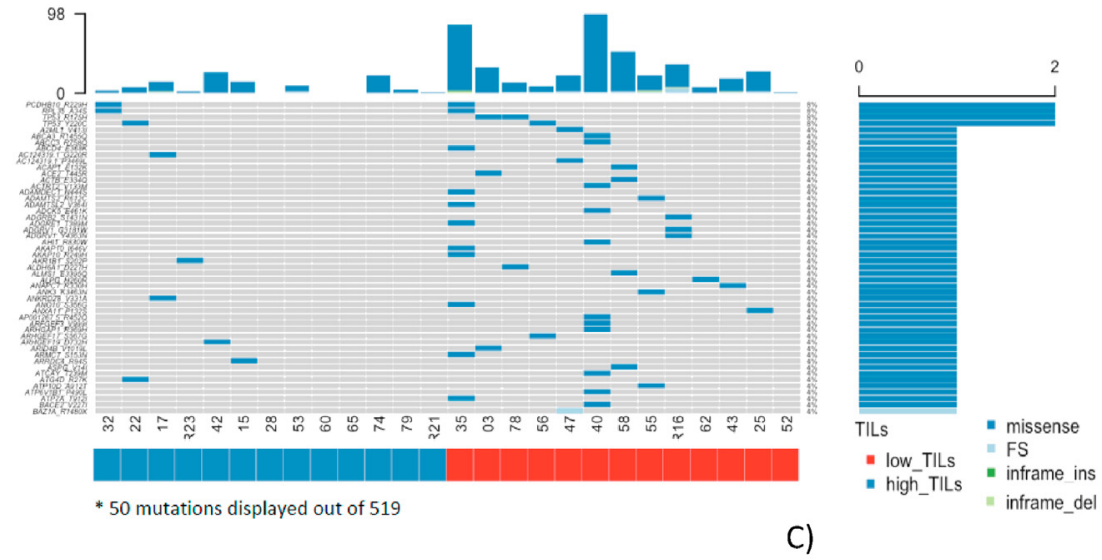

B)

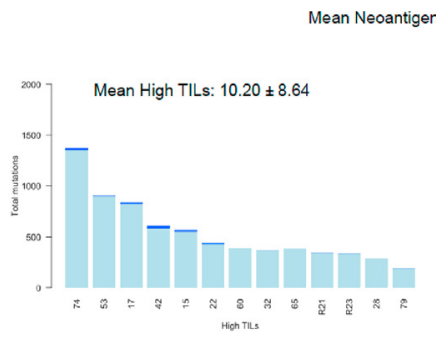

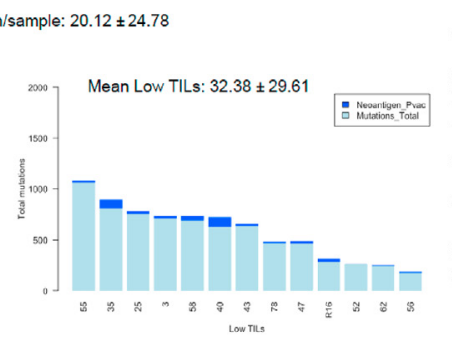

C)

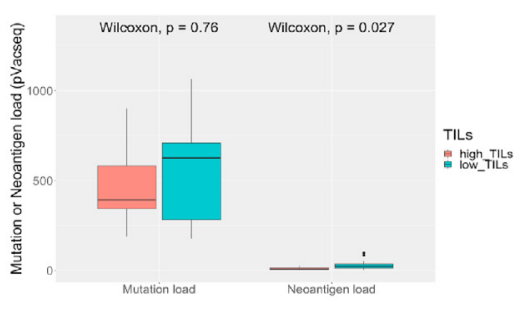

Fig. 5. Results of whole exome sequencing analysis. a) Point mutations with potential to generate neoantigens (displayed 50 of 519 ); b) mutations, neoantigens and neoantigen mean of high TILs and low TIL patients; c) mutation and neoantigen load comparison between high and low TIL patients. FS, frame shift; inframe_ins/del, inframe insertion/deletion.

$20.12 \pm 24.78$; high TILs had $10.20 \pm 8.64$ and low TILs $32.38 \pm 29.6$ (Fig. 5b). Low TILs had more nonsignificant median mutation load $(\mathrm{p}=0.76)$ and significant neoantigen load ( $\mathrm{p}=0.03$ ) (Fig. 5c).

\section{Discussion}

Understanding how the immune system interacts with tumour cells is key to develop successful therapeutic strategies. In TNBC, high levels of TILs have been correlated with prognosis and response to chemotherapy [5-13]. However, the reason why some tumours have a large lymphocyte infiltration and others do not is a matter of in-depth research. It is well known that inhibition or activation, proliferation and migration of immune cells is controlled through immune checkpoints, cytokines and other molecules [25]. If we could understand the mechanisms underlying poor immune infiltration, we could apply treatments to interfere with it and provoke the migration of the immune cells to the tumour to exert their action.

We have demonstrated that, in a group of TNBC patients with very low or zero TILs, T cells seem to be deactivated and get retained in local LN by, at least, CTLA-4, in comparison to a group of patients with high levels of TILs. We also found that these patients overexpress B7.H3 and B7.H4 in the tumour. In high TILs, we observed more expanded humoral response, more PD-L1+ tumour cells and less neoantigen load.
Only few researchers have previously analysed the immune cell subtypes in $\mathrm{LN}$ of breast cancer patients [15-18]. LN have mostly been studied to correlate with tumour progression as they are usually the first place of metastasis. In addition, LNs are of extreme importance in cancer immunity because it is where antigen presentation and lymphocyte proliferation occur to respond to tumour first appearance. As commented before, we have observed higher expression of CTLA-4 in TNBC patients with low numbers of TILs, that may cause anergy in axillary lymphocytes, preventing their response to immune attraction and migration to tumour bed. CTLA-4 is an immune checkpoint with a key role in early inhibition of cellular responses. We also observed higher expression of OX-40 in the LN of these patients, which is a co-stimulatory molecule that appears later in the immune response to maintain lymphocyte activation. These results suggest that we could use anti-CTLA4 drugs to revive and "liberate" those lymphocytes that have recognised tumour antigens and induced their migration to the tumour. This is something that in our opinion should be explored in future clinical studies. Only the ULTIMATE phase II trial (NCT02997995) is exploring this strategy, but in ER+/HER2- (estrogen receptor +/human epidermal growth factor receptor 2-) patients.

OX-40 was expressed in the cytoplasm and membrane of tumour cells, and low TIL patients overexpressed this protein in compare to high TIL patients. 
OX-40 expression has been mainly studied in TILs, so their role in tumour cells is unclear and we could not conclude anything from this finding. Gene expression results revealed that B7.H3, B7.H4, VEGF, CD56 and CD73 were highly expressed in low TILs. We thought that B7.H3 and B7.H4 were probably present on tumour cells as these patients lack immune infiltration. Another group has recently published similar results when measuring B7.H3 and B7.H4 not only by gene expression but also by RNAscope ISH (in situ hybridisation) and IHC [26]. They saw an inverse correlation between these two genes and stromal CD3 and CD8 infiltration; and that these markers were mainly expressed in tumour cells as seen by IHC. Both receptors are from the B7 family, like PD-L1 (B7.H1) and PD-L2 (B7-DC), but their ligands are unknown [27]. We hypothesised that tumour cells of low TIL patients may be using these immune checkpoint inhibitors to avoid immune infiltration, although $\mathrm{B} 7 . \mathrm{H} 3$ have a co-stimulatory role also. As they have precisely low immune infiltration, it is difficult to think how they may inhibit $\mathrm{T}$ cells, as it is necessary direct contact between receptors and ligands. They may be inhibiting natural killer cells (CD56+), which are very important cells for the innate response to control tumours, and were overrepresented in low TILs patients. Higher expression of VEGF in low TIL patients may indicate a rapid formation of new vessels because their tumour cells grow faster than in high TILs patients, which may be due to the lack of immune surveillance over them. CD73 is an ectonucleotidase with immunosuppressive effects that is usually expressed together with CD39.

The 50-immune gene panel showed that LN of high TILs patients had an overrepresentation of CD68 and CD8 or CD4 cells based on two different statistical analysis. It might suggest more antigen presentation by macrophages (probably apoptotic rests of tumour cells due to cytotoxic $\mathrm{T}$ cell activity), and more $\mathrm{T}$ cell proliferation in those patients, which can provide a larger specific response to tumour neoantigens. We could infer that the immune response has been partially effective, and the problem may lie in the in-situ deactivation of lymphocytes by PD-L1, which is expressed in tumour cells and adjacent stromal lymphocytes of high TILs. And it seems that the negative feedback first starts in the immune infiltrate, and after in tumour cells, as seen in the binary PD-L1 comparison and in other studies $[20,28]$. We noticed OX-40+ TILs were mostly surrounding tumour nests, which could be tumour-specific $\mathrm{T}$ helper cells as previously described $[18,28]$; the same occurred with PD-L1+ and CD8 $+\mathrm{T}$ cells, which were adjacent to tumour. Based on this, we could hypothesise that the cytotoxic $T$ cells may be receiving a negative feedback from $T$ regulatory cells near the tumour. Therefore, in high TILs, we could apply anti-PD-(L)1 drugs to activate those lymphocytes already present within the tumour. Other ICIs like anti-LAG3 or anti-
TIM3 may be also considered as they were overexpressed based on gene expression results. We also noticed that HLA-E was overrepresented in high TILs, which is a characteristic of the altered HLA tumour phenotype VII, together with low or null expression of classical HLA class I molecules [29]. This phenotype allows tumour cells to escape $\mathrm{T}$ cell and NK immune attack as they have reduced ability to present tumour antigens in their surface. In low TILs, stroma signature was upregulated, being extensive and stiff stroma, a defensive way to evade immune infiltration, allowing tumour cells to grow faster and, therefore, achieving a hypoxia state that requires angiogenesis. Although TGF-beta signature in the forest plot showed a trend to be more expressed in high TILs (being one of those TGFB1), the SAM analysis showed that low TILs had several upregulated genes of this signature (including TGFB2) and none downregulated in comparison to high TIL patients.

We performed WES analysis to analyse the correlation between number of TILs with tumour mutations and neoantigens. Interestingly, we observed that high TILs had lower median mutations and statistically significant less neoantigens. Karn et al. [30] obtained similar results, suggesting that immune cells may eliminate immunogenic tumour clones (immune surveillance), resulting in lower clonal tumour heterogeneity of high TILs patients and, therefore, less neoantigens. However, we could not rule out that if higher number of neoantigens might induce a potent immune response with highly effective lymphocyte activation first and important inhibition by CTLA-4 after in LN of low TILs. This is why we also hypothesised that, in high TILs, several immunogenic tumour clones may have been in the beginning at higher numbers, inducing a fully $\mathrm{T}$ cell response and being completely eliminated. Low TILs may have had more immunogenic different clones but under-represented in quantity, not being able to complete a fully activation of the $\mathrm{T}$ cells. We also consider the possibility that $\mathrm{T}$ cells present in the tumour of high TIL patients might have encountered those neoantigens before from a previous microscopic and less heterogenic tumour that was completely eradicated; or if those neoantigens share the sequence with other nontumour antigens that previously elicited an immune response in the patient.

The study of the GC and TLS shed some light about the role of humoral response in cancer. We observed that high TILs tended to have more presence of TLS in the tumour, which was similar to other studies [31], and were more numerous and larger, providing a specific and faster in situ humoral response to cancer growth. But, it was also surprising that some low TIL patients, with no tumour immune infiltration, were able to develop a specific humoral response at the periphery of the tumour. In LN of high TIL patients, we observed more and larger GCs by IHC, but we did not see a statistically 
significant overexpression of B cell markers with the NanoString panel. This may be due to the difference in the technique, or because of the presence of the $\mathrm{GC}$-specially the smaller ones-depends on the cut section.

Spitzer et al. [32] studied the local and systemic immunity in mice treated with an effective immunotherapy (alloIgG + anti-CD40 + IFN- $\gamma$ ) in comparison to an ineffective therapy (anti-PD-1) or no treatment. The authors observed an increase of CD4 and CD8 T cells in $\mathrm{LN}$ during priming and tumour rejection, which is the same effect observed in the high TIL group. They also noticed an expansion of activated naïve $B$ cells expressing immunoglobulin $\mathrm{M}$ but low levels of immunoglobulin D. We did not detect an increase of B cell gene counts: they were equally and highly expressed in the $\mathrm{LN}$ of both groups. Other important finding of their study was the activation of the systemic immune response and in organs that are far from the tumour, like the spleen, during priming; and with a decrease during tumour rejection, which may reflect immune cells moving into the tissues. We agree that, after antigen presentation and activation in nearby LNs, those T cells are released systemically; not only to migrate to the tumour site but also to look for potential cancer cells that have entered in the blood stream or settled down in another organ.

Finally, we noticed that high TILs patients tended to be younger. If it was by chance, because of the low number of patients analysed, or it was because the immune system in young people is more reactive, cannot be demonstrated with this study. However, this has been observed in other studies with a larger sample size [33].

This study has several limitations. First, the sample size was very limited; however, high TIL patients are approximately $15 \%$ of all TNBC patients, and TNBC patients usually have one or more LN with metastases at diagnosis. In addition, many of these patients receive neoadjuvant chemotherapy. Second, we cannot be sure about the sentinel LN in patients who underwent lymphadenectomy, so we had to decide that choosing one from the first level and with the highest number of GCs was a fair approximation. Third, in some LN, it was difficult to distinguish the different parts, so in those antibodies that stained different cells, it was not possible to score only lymphocytes in the paracortex. We could score lymphocytes only with CTLA-4 and PD-1 antibodies, which were the principal immune checkpoints to test the main hypothesis. Forth, it would have been interesting to have LN of healthy women as controls, to assess how these two groups behave in comparison to normal physiological conditions, as done in other studies [15].

In conclusion, we demonstrated that lymphocytes could be deactivated by, at least, CTLA-4 in tumour- draining LN of TNBC patients with low number of TILs, which prevents them to respond to other immune stimuli and, therefore, they get retained in the LN. Thus, exploring new strategies to revive and release these lymphocytes might have important clinical implications.

\section{Author contributions}

J.C. conceived the experimental design and designed the inclusion and exclusion criteria for the study, identified research subjects and obtained samples. A.Q., E.M.-C. and A.P. provided input into the experimental design. A.Q. and V.P. identified research subjects, obtained samples, optimised antibodies, evaluated TILs and antibodies under the microscope, and interpreted the data. T.M. assisted with antibody optimisation, sample preparation and processing. G.V. and R.D. provided input for sample size, analysed and interpreted the data and designed figures. J.C. and A.Q. designed the 50immune gene NanoString panel and contracted vendors to run this panel and WES. A.P., L.P. and P.G. run the $360^{\circ}$ NanoString Breast Cancer panel, analysed and interpreted the data from both NanoString panels and designed figures. J.B.-H., C.A., M.V. and L.M.-A. developed the pipelines for mutation calling and neoantigen prediction, analysed and interpreted the sequencing data and designed figures. A.Q., L.M.-A and J.C. wrote the manuscript. P.S., G.C., M.M. and J.P.-G. assisted with manuscript writing.

\section{Financial support}

This project was supported by a grant from INTHEOS Foundation, thanks to the donation received from Pompadour, and from FERO Foundation.

\section{Conflict of interest statement}

The authors declare the following financial interests/ personal relationships, which may be considered as potential competing interests: A.Q. received transportation and accommodation fees from MSD outside the submitted work. V.P. has received fees as consultant, participated in advisory boards or received travel grants from Sysmex, Roche, MSD, AstraZeneca and Genomic Health. A.P. has received personal fees from Roche, Pfizer, Novartis, Amgen, BMS, Nanostring Technologies and Daiichi Sankyo; fees as a consultant from Roche, Pfizer, Novartis, Amgen, BMS, Puma, Oncolytics Biotech, MSD and Lilly; grants from Boehringer, Novartis, Roche, Nanostring, Sysmex Europa GmbH, MedSIR, Celgene, Astellas and Pzifer; is a member of the executive board of Reveal Genomics, SL, Beast International Group (BIG) and SOLTI cooperative group; and in the patronage committee of SOLTI Foundation and Actitud Frente al Cáncer Foundation. R.D. has 
declared advisory role for Roche, Boehringer Ingelheim, has received a speaker's fee from Roche, Ipsen, Amgen, Servier, Sanofi, Merck Sharp \& Dohme, and research grants from Merck and Pierre Fabre. P.S. has received personal fees and fees as a consultant from AstraZeneca, Bayer, Boehringer Ingelheim, Merck, Novartis, Pfizer, Puma and Roche; grants from Astellas, AstraZeneca, Genentech, Novartis, Oncogenex and Roche. C.G. reports grants from Roche and Pfizer; and received personal fees from Daichii Sankyo, MSD, Astra Zeneca, outside the submitted work. J.P-G. has received fees as a consultant from Roche and Eli Lilly; and travel and accommodation expenses from Roche. E.M-C. has received travel and accommodation fees from BMS and MSD; personal fees and fees as a consultant from Novartis, Roche, BMS, MSD, Pier Fabre and Sanofi. L.M-A. has received consultancy from Roche; received an educational grant and travel from BMS; holds a research collaboration with NanoString Technologies; and received grants to the Institution from Grifols, Gilead, MSD, Jansen and AbbVie. J.C. has received fees as a consultant from Roche, Celgene, Cellestia, AstraZeneca, Biothera Pharmaceutical, Merus, Seattle Genetics, Daiichi Sankyo, Erytech, Athenex, Polyphor, Lilly, Servier, MSD, GSK, Leuko; personal fees from Roche, Novartis, Celgene, Eisai, Pfizer, Samsung Bioepis, Lilly, MSD, Daiichi Sankyo; research funding from Roche, Ariad pharmaceuticals, AstraZeneca, Baxalta GMBH/Servier Affaires, Bayer healthcare, Eisai, F. Hoffman-La Roche, Guardanth health, MSD, Pfizer, Piqur Therapeutics, Puma C, Queen Mary University of London, Seagen; and owns stock, patents and intellectual property from MedSIR. T.M., G.V., L.P., P.G., M.M., J.B.-H., C.A. and M.V. declare no competing interests.

\section{Acknowledgements}

We want to particularly acknowledge the patients and the BioBank Hospital Ramón y Cajal-IRYCIS (PT13/0010/0002), integrated in the Spanish National Biobanks Network. We would also want to acknowledge INTHEOS and FERO Foundations from their financial support.

\section{Appendix A. Supplementary data}

Supplementary data to this article can be found online at https://doi.org/10.1016/j.ejca.2021.01.037.

\section{References}

[1] Schmid P, Adams S, Rugo HS, Schneeweiss A, Barrios CH, Iwata $\mathrm{H}$, et al. Atezolizumab and nab-paclitaxel in advanced triple-negative breast cancer. N Engl J Med 2018;379(22): $2108-21$.
[2] Schmid P, Rugo HS, Adams S, Schneeweiss A, Barrios CH, Iwata $\mathrm{H}$, et al. Atezolizumab plus nab-paclitaxel as first-line treatment for unresectable, locally advanced or metastatic triplenegative breast cancer (IMpassion130): updated efficacy results from a randomised, double-blind, placebo-controlled, phase 3 trial. Lancet Oncol 2019;1:44-59.

[3] Cortes J, Cescon David W, Rugo Hope S, Nowecki Zbigniew, Seock-Ah Im, Mastura Md Yusof, et al. KEYNOTE-355: randomized, double-blind, phase III study of pembrolizumab + chemotherapy versus placebo + chemotherapy for previously untreated locally recurrent inoperable or metastatic triplenegative breast cancer. J Clin Oncol 2020;38(15_suppl):1000.

[4] Schmid P, Cortes J, Pusztai L, McArthur H, Kümmel S, Bergh J, et al. Pembrolizumab for early triple-negative breast cancer. N Engl J Med 2020;382(9):810-21.

[5] Loi S, Sirtaine N, Piette F, Salgado R, Viale G, Van Eenoo F, et al. Prognostic and predictive value of tumor-infiltrating lymphocytes in a phase III randomized adjuvant breast cancer trial in node-positive breast cancer comparing the addition of docetaxel to doxorubicin with doxorubicin-based chemotherapy: BIG 0298. J Clin Oncol 2013;31(7):860-7.

[6] Loi S, Michiels S, Salgado R, Sirtaine N, Jose V, Fumagalli D, et al. Tumor infiltrating lymphocytes are prognostic in triple negative breast cancer and predictive for trastuzumab benefit in early breast cancer: results from the FinHER trial. Ann Oncol 2014;25(8):1544-50.

[7] Loi S, Drubay D, Adams S, Pruneri G, Francie P, et al. Tumorinfiltrating lymphocytes and prognosis: a pooled individual patient analysis of early-stage triple-negative breast cancers. J Clin Oncol 2019;37(7):559-69.

[8] Denkert C, Loibl S, Noske A, Roller M, Müller BM, Komor M, et al. Tumor-associated lymphocytes as an independent predictor of response to neoadjuvant chemotherapy in breast cancer. J Clin Oncol 2010;28(1):105-13.

[9] West NR, Milne K, Truong PT, Macpherson N, Nelson BH, Watson PH. Tumor-infiltrating lymphocytes predict response to anthracycline-based chemotherapy in estrogen receptor-negative breast cancer. Breast Cancer Res 2011;13(6):R126.

[10] Denkert C, von Minckwitz G, Brase JC, Sinn BV, Gade S, Kronenwett R, et al. Tumor-infiltrating lymphocytes and response to neoadjuvant chemotherapy with or without carboplatin in human epidermal growth factor receptor 2-positive and triple-negative primary breast cancers. J Clin Oncol 2015;33(9): 983-91.

[11] Issa-Nummer $\mathrm{Y}$, Darb-Esfahani S, Loibl S, Kunz G, Nekljudova V, Schrader I, et al. Prospective validation of immunological infiltrate for prediction of response to neoadjuvant chemotherapy in HER2-negative breast cancer-a substudy of the neoadjuvant GeparQuinto trial. PloS One 2013;8(12):e79775.

[12] Adams S, Gray RJ, Demaria S, Goldstein L, Perez EA, Shulman LN, et al. Prognostic value of tumor-infiltrating lymphocytes in triple-negative breast cancers from two phase III randomized adjuvant breast cancer trials: ECOG 2197 and ECOG 1199. J Clin Oncol 2014;32(27):2959-66.

[13] Loi S, Drubay D, Adams S, Francis PA, Joensuu H, Dieci MV, et al. Abstract S1-03: pooled individual patient data analysis of stromal tumor infiltrating lymphocytes in primary triple negative breast cancer treated with anthracycline-based chemotherapy. Canc Res 2016;76(4 Suppl):S1-03. Abstract nr.

[14] Stanton SE, Adams S, Disis ML. Variation in the incidence and magnitude of tumor-infiltrating lymphocytes in breast cancer subtypes: a systematic review. JAMA Oncol 2016;2:1354-60.

[15] Kohrt HE, Nouri N, Nowels K, Johnson D, Holmes S, Lee PP. Profile of immune cells in axillary lymph nodes predicts diseasefree survival in breast cancer. PLoS Med 2005;2(9):e284.

[16] Seidl M, Bader M, Vaihinger A, Wellner UF, Todorova R, Herde B, et al. Morphology of immunomodulation in breast 
cancer tumor draining lymph nodes depends on stage and intrinsic subtype. Sci Rep 2018;8(1):5321.

[17] Poindexter NJ, Sahin A, Hunt KK, Grimm EA. Analysis of dendritic cells in tumor-free and tumor-containing sentinel lymph nodes from patients with breast cancer. Breast Cancer Res 2004; 6(4):R408-15.

[18] Ramstad T, Lawnicki L, Vetto J, Weinberg A. Immunohistochemical analysis of primary breast tumors and tumor-draining lymph nodes by means of the T-cell costimulatory molecule OX-40. Am J Surg 2000;179(5):400-6.

[19] Hendry S, Salgado R, Gevaert T, Russell PA, John T, Thapa B, et al. Assessing tumor-infiltrating lymphocytes in solid tumors: a practical review for pathologists and proposal for a standardized method from the international immunooncology biomarkers working group: Part 1: assessing the host immune response, TILs in invasive breast carcinoma and ductal carcinoma in situ, metastatic tumor deposits and areas for further research. Adv Anat Pathol 2017;24(5):235-51.

[20] Tawfik O, Kimler BF, Karnik T, Shehata P. Clinicopathological correlation of PD-L1 expression in primary and metastatic breast cancer and infiltrating immune cells. Hum Pathol 2018;80:170-8.

[21] NanoString Technologies. nCounter ${ }^{\circledR}$ Breast Cancer 360TM Panel. Available at: https://www.nanostring.com/products/geneexpression-panels/gene-expression-panels-overview/ncounterbreast-cancer-360-panel. [Accessed 8 July 2020].

[22] Cibulskis K, Lawrence MS, Carter SL, Sivachenko A, Jaffe D, Sougnez C, et al. Sensitive detection of somatic point mutations in impure and heterogeneous cancer samples. Nat Biotechnol 2013; 31(3):213-9.

[23] Jurtz V, Paul S, Andreatta M, Marcatili P, Peters B, Nielsen M. NetMHCpan-4.0: improved peptide-MHC class I interaction predictions integrating eluted ligand and peptide binding affinity data. J Immunol 2017;199(9):3360-8.
[24] Hundal J, Carreno BM, Petti AA, Linette GP, Griffith OL, Mardis ER, et al. pVAC-Seq: a genome-guided in silico approach to identifying tumor neoantigens. Genome Med 2016;8(1):11.

[25] Kruger S, Ilmer M, Kobold S, Cadilha BL, Endres S, Ormanns S, et al. Advances in cancer immunotherapy 2019 - latest trends. J Exp Clin Canc Res 2019;38(1):268.

[26] Kim NI, Park MH, Kweon SS, Lee JS. B7-H3 and B7-H4 expression in breast cancer and their association with clinicopathological variables and T cell infiltration. Pathobiology 2020; 87(3):179-92.

[27] Ni L, Dong C. New B7 family checkpoints in human cancers. Mol Canc Therapeut 2017;16(7):1203-11.

[28] Sobral-Leite M, Van de Vijver K, Michaut M, van der Linden R, Hooijer GKJ, Horlings HM, et al. Assessment of PD-L1 expression across breast cancer molecular subtypes, in relation to mutation rate, $\mathrm{B} R C A 1$-like status, tumor-infiltrating immune cells and survival. OncoImmunology 2018;7(12):e1509820.

[29] Garrido F. MHC/HLA class I loss in cancer cells. Adv Exp Med Biol 2019;1151:15-78.

[30] Karn T, Jiang T, Hatzis C, Sänger N, El-Balat A, Rody A, et al. Association between genomic metrics and immune infiltration in triple-negative breast cancer. JAMA Oncol 2017;3(12):1707-11.

[31] Buisseret L, Garaud S, de Wind A, Van den Eynden G, Boisson A, Solinas C, et al. Tumor-infiltrating lymphocyte composition, organization and PD-1/PD-L1 expression are linked in breast cancer. OncoImmunology 2016;6(1):e1257452.

[32] Spitzer MH, Carmi Y, Reticker-Flynn NE, Kwek S, Madhireddy D, et al. Systemic immunity is required for effective cancer immunotherapy. Cell 2017;168(3):487-502.

[33] Denkert C, von Minckwitz G, Darb-Esfahani S, Lederer B, Heppner BI, Weber KE, et al. Tumour-infiltrating lymphocytes and prognosis in different subtypes of breast cancer: a pooled analysis of 3771 patients treated with neoadjuvant therapy. Lancet Oncol 2018;19(1):40-50. 